\title{
Participation of vasoactive intestinal polypeptide in ovarian steroids production during the rat estrous cycle and in the development of estradiol valerate-induced polycystic ovary
}

\author{
Claudio Parra, Jenny L Fiedler, S Leticia Luna ${ }^{1}$, Monika Greiner, Vasantha Padmanabhan ${ }^{2}$ \\ and Hernán E Lara
}

\begin{abstract}
Lab. Neurobioquímica, Departamento de Bioquímica y Biología Molecular, Facultad de Ciencias Químicas y Farmacéuticas, Universidad de Chile, Santiago, Chile, ${ }^{1}$ Facultad de Química y Farmacia, Universidad de Valparaíso and ${ }^{2}$ Department of Pediatrics and Reproductive Sciences Program, University of Michigan, Ann Arbor, Michigan, USA
\end{abstract}

Correspondence should be addressed to H E Lara, Department of Biochemistry and Molecular Biology, Faculty of Chemistry and Pharmaceutical Sciences, Universidad de Chile, PO Box 233, Santiago, Chile; Email: hlara@ciq.uchile.cl

C Parra is now at Universidad Iberoamericana de Ciencias y Tecnología

\begin{abstract}
Vasoactive intestinal polypeptide (VIP) stimulates estradiol and progesterone release from ovarian granulosa cells in vitro. Very little information is available as to the role VIP plays in the control of steroid secretion during reproductive cyclicity and in ovarian pathologies involving altered steroid secretion. In this study, we determined the involvement of VIP in regulating ovarian androgen and estradiol release during estrous cyclicity and estradiol valerate (EV)-induced polycystic ovarian development in rats. Our findings show that androgen and estradiol release from ovaries obtained during different stages of rat estrous cycle mimic cyclic changes in steroid release observed in vivo with maximal release occurring during late proestrus. VIP increased androgen release from ovaries of all cycle stages except late proestrus and estradiol release from all cycle stages. Increases in VIP-induced androgen and estradiol release were maximal at early proestrus. Inclusion of saturating concentrations of androstenedione increased magnitude of VIP-induced estradiol release at diestrus and estrus but not proestrus. Magnitude of VIP-induced androgen and estradiol release tended to be greater in the ovaries from EV-treated rats with polycystic ovary compared with estrous controls. At the tissue level, ovarian VIP concentration was cycle stage dependent with highest level seen in diestrus. Maximum concentration of VIP was found in EV-treated rats. Changes in VIP were inversely related to changes in ovarian nerve growth factor, a neuropeptide involved in ovarian androgen secretion. These results strongly suggest that intraovarian VIP participates in the control of estradiol secretion during the rat estrous cycle and possibly in the maintenance of increased ovarian estradiol secretory activity of EV-treated rats.
\end{abstract}

Reproduction (2007) 133 147-154

\section{Introduction}

The ovary, in addition to the endocrine and intraovarian control, is regulated by direct neural inputs of both catecholaminergic (Klein \& Burden 1988, Burden 1998) and peptidergic nature (Ojeda et al. 1989, Ojeda \& Urbanski 1994). Previous studies have found that norepinephrine (NE) acts on $\beta_{2}$-adrenergic receptors present in theca and granulosa cells and stimulates progesterone $\left(\mathrm{P}_{4}\right)$ and androgens but not estradiol secretion (Ojeda \& Lara 1989). In contrast to NE, vasoactive intestinal peptide (VIP), another neuropeptide found in ovarian nerves, stimulates estradiol and $\mathrm{P}_{4}$ release from cultured granulosa cells and whole ovaries in vitro (Davoren \& Hsueh 1985, Ahmed et al. 1986), $\mathrm{P}_{4}$ release in vivo (Fredericks et al. 1983), and androgen release from ovarian fragments in vitro (Ahmed et al. 1986). These effects may be related to the ability of VIP to enhance the synthesis of the cholesterol side-chain cleavage enzyme complex (Trzeciak et al. 1986), the rate-limiting step in $\mathrm{P}_{4}$ biosynthesis and the activity of the aromatase enzyme complex (George \& Ojeda 1987). In addition, in the neonatal ovary, VIP also induces folliclestimulating hormone $(\mathrm{FSH})$ receptor and aromatase mRNA and protein expression several days before the ovary becomes responsive to gonadotropins (Mayerhofer 
et al. 1997). These findings suggest that VIP may play a vital role in the acquisition of cyclic ovarian function.

Although existing evidence suggests a role for VIP in the control of ovarian steroidogenesis, it is unclear to what extent VIP is involved in the maintenance of the cyclic steroid production. If VIP as a neuropeptide participates in cyclic steroid production, the activation of VIPergic nerves is likely to be cyclic in nature and/or the sensitivity of the ovary to VIP will change with stage of reproductive cycle or development of ovarian pathologies. Previously, we found an increase in norepinephrine-dependent androgen and $\mathrm{P}_{4}$ secretion and a norepinephrine-independent estradiol secretion from estradiol valerate (EV)-induced polycystic ovaries in rats (Rosa-E-Silva et al. 2003). A possibility to consider is that VIP, because of its ability to stimulate estradiol secretion from the ovary, may be responsible for the observed increase in estradiol production in this model of ovarian pathology. In this study, we tested the hypothesis that VIP content and/or ovarian sensitivity to VIP are positively related to changes in ovarian androgens and estradiol release during the rat estrous cycle and the development of EV-induced polycystic ovarian development.

\section{Materials and Methods}

\section{Animals}

Virgin adult cycling Sprague-Dawley rats (200-220 g, $60 \pm 5$ days of age) were obtained from a stock maintained at the University of Chile. They were allowed free access to pelleted food and tap water and were housed two rats per cage under controlled temperature $\left(22{ }^{\circ} \mathrm{C}\right.$ ) and photoperiods (lights on from 0700 to $1900 \mathrm{~h}$ ). Only animals exhibiting regular 4-day estrous cycles (monitored by daily vaginal smears obtained between 1000 and $1200 \mathrm{~h}$ from 60 to 120 days of age) were used in these studies. Three sets of studies were undertaken. The first set of studies were carried out when the rats were approximately 4-month old and focused on VIP regulation of ovarian androgen and estrogen secretion during the rat cycle. Androgen and estrogen release from ovaries procured at various time points during the rat estrus cycle (diestrus (DII), $n=6$; early proestrus (EP), $n=5$; late proestrus (LP), $n=6$; and estrus (E), $n=5)$ were examined both in the absence or presence of $10^{-4} \mathrm{M}$ saturating concentration of androstenedione. The choice of androstenedione was based on earlier studies that found inclusion of increasing concentrations of androstenedione increases estradiol in a dose-dependent manner (Hillier \& De Zwart 1981, Adashi \& Hsueh 1982). Saturating levels of androstenedione were used to overcome any reduction in substrate availability and to gain a preliminary understanding of aromatase availability. The difficulty associated with precisely timing metestrus, as it lasts less than a day in our group of rats, precluded us from including this stage of the estrous cycle in this study.

The second study addressed the effects of VIP on androgen and estrogen release from polycystic ovaries of EV-treated rats $(n=5)$. Rats were injected with $2 \mathrm{mg}$ EV on day $60 \pm 5$ of postnatal life and studied 60 days later. In previous studies, we have demonstrated that this mode of treatment leads to polycystic ovarian morphology, persistent estrus condition accompanied with increased circulating estradiol levels (Brawer et al. 1986, Lara et al. 1993), and hypersecretion of androgens and estradiol from ovaries incubated in vitro (Brawer et al. 1986, Barria et al. 1993, Lara et al. 1993, Rosa-E-Silva et al. 2003). Anovulation combined with hyperandrogenism and/or polycystic ovaries are features reflective of women with poly cystic ovary syndrome (PCOS) (Rotterdam consensus 2004). Since EV-treated rats are in constant estrus as monitored by daily vaginal lavages, estrus females $(n=5)$ of similar age were studied in parallel as controls. Five other EV-treated animals were processed for ovarian morphology to establish if the EV-treatment generated the expected phenotype.

The third study determined cyclic changes in ovarian concentrations of VIP, NE, and nerve growth factor (NGF) in DII, EP, and $E$ rats and compared it with that of EV-treated rats ( $n=5 /$ treatment group). With the exception of LP rats, which were killed at $1700 \mathrm{~h}$, all other animals were terminated between 1000 and $1200 \mathrm{~h}$. After decapitation, ovaries were rapidly removed; those intended for measuring in vitro steroid release were immediately transferred to Krebs-bicarbonate buffer; those for VIP, NE, and NGF content were frozen at $-80{ }^{\circ} \mathrm{C}$. Since preliminary experiments showed no differences in measures of NE and VIP between the left and the right ovary, we used one ovary for NE and the other for VIP determination. To correlate the changes in the capacity of the ovary to release steroids by VIP or NE, we analyzed both neurotransmitters in the ovary as well as of NGF, the neuropeptide involved in the development of the NE-induced hyperandrogenic condition during polycystic ovary. All animal procedures were performed using protocols previously approved by the Institutional Ethic Committee of Faculty of Chemical and Pharmaceutical Sciences, Universidad de Chile and experiments were conducted in accordance with the International Guiding Principles for Biomedical Research.

\section{Steroid response to VIP stimulation}

Ovaries were halved and incubated in $2 \mathrm{ml}$ KrebsRinger bicarbonate buffer $(\mathrm{pH} 7.4)$, for $3 \mathrm{~h}$ at $37{ }^{\circ} \mathrm{C}$ as previously described (Barria et al. 1993). The ovaries were incubated in the absence (one ovary) or presence of $10^{-6}$ M VIP (Sigma Chem. Co; one ovary). The concentration of VIP was chosen to produce maximal estradiol secretion from the rat ovary, on the basis of previous studies (Ahmed et al. 1986). The incubation 
time was chosen from preliminary studies to be in the linear phase of release. Androgen (A) and estradiol released into the incubation medium were measured by previously described radioimmunoassays (Barria et al. 1993). The sensitivity, intra-, and interassay coefficient of variation of the estradiol assays were $3.9 \mathrm{pg}, 7$, and $12 \%$ respectively. Cross-reactivity of androstenedione in estradiol assay is $<0.001 \%$ (England et al. 1974). The sensitivity, intra-, and interassay coefficient of variation of the androgen assay were $1.5 \mathrm{pg}, 5$, and $9 \%$ respectively. Since the antiserum employed (GDN-250) not only detects testosterone but also cross-reacts with $5 \alpha$-dihydrotestosterone, the values are reported as androgen (Advis \& Ojeda 1978).

\section{Biochemical measures}

One ovary of each rat was used for NGF determination and the other was used for VIP and NE content. NE and NGF measurements were undertaken as positive controls to confirm previous findings of sympathetic input.

\section{NGF quantification}

The NGF E-max Immunoassay system (Promega) was used to quantify ovarian NGF content as previously described (Dissen et al. 2000, Dorfman et al. 2003). The ovaries were weighed, homogenized in 1:4 Dulbecco's PBS (pH 7.35), and centrifuged for 15 min (13 000 r.p.m.). ELISA 96-well plates were coated overnight at $4{ }^{\circ} \mathrm{C}$ with anti-NGF polyclonal antibody (pAb; 1:1000; $100 \mu \mathrm{l} /$ well). Following day, the plates were washed and incubated at room temperature (RT) for $1 \mathrm{~h}$ with block and sample buffer. Ovarian samples (50, 100, and $200 \mu$ adjusted to a final volume of $200 \mu \mathrm{l}$ ) were added to each well and the plate incubated for $6 \mathrm{~h}$. The plates were then washed and incubated overnight at $4{ }^{\circ} \mathrm{C}$ with anti-NGF mAb and they were again washed and incubated with anti-rat IgG-HRP (horse radish peroxidase) conjugate for $2.5 \mathrm{~h}$ at room temperature. After this incubation, TMB One solution (tetramethylbenzidine and the peroxidase substrate) was added to each well, and the plates were shaken for 5-10 min at RT; adding $1 \mathrm{M}$ hydrochloric acid to the wells terminated the reaction. The optical density of the reaction product was read on a microplate reader at $450 \mathrm{~nm}$ and the values were normalized per milligram of tissue assayed.

\section{NE determination}

The other ovary was homogenized in $200 \mu \mathrm{l}$ of $0.1 \mathrm{M}$ acetic acid. The homogenate $(10 \mu \mathrm{l})$ was precipitated with $180 \mu \mathrm{l} 0.2 \mathrm{M} \mathrm{HClO}_{4}$ and centrifuged $(15000 \mathrm{~g}$, $15 \mathrm{~min}$ ). The supernatant was used for NE determination by HPLC with electrochemical detection (Dorfman et al. 2003). A $150 \mu \mathrm{l}$ sample was mixed with $50 \mathrm{mg}$ activated alumina in $1 \mathrm{ml}$ Tris $1.5 \mathrm{M}(\mathrm{pH} 8.3-8.5)$ and $\%$ EDTA. Dihydroxybenzyl amine (DHBA, 2000 pg in $20 \mu \mathrm{l}$ ) was added as an internal standard. The alumina was rinsed thoroughly with nanopure water and NE was eluted with $100 \mu \mathrm{l}$ of $0.2 \mathrm{M}$ perchloric acid and centrifuged; $20 \mu \mathrm{l}$ resulting supernatant was injected into a Waters HPLC system with a C18 reverse phase column (Lichrosphere, 60 RP-Select B, Merck) and an electrochemical detector (Waters 464). The mobile phase contained $0.1 \mathrm{M}$ $\mathrm{NaH}_{2} \mathrm{PO}_{4}, 0.42 \mathrm{mM}$ octyl sulfate, $0.02 \%$ EDTA, and $1.5 \%$ acetonitrile $(\mathrm{pH} 2.5)$ with a flow rate of $0.9 \mathrm{ml} / \mathrm{min}$. The potential of the amperometric detector was set at $0.7 \mathrm{~V}$. Under these experimental conditions, the retention time was $4 \mathrm{~min}$ for $\mathrm{NA}$ and $10 \mathrm{~min}$ for the DHBA used to correct for procedural recovery.

\section{VIP determination}

The remaining $190 \mu \mathrm{l}$ homogenate from the above was boiled at $100{ }^{\circ} \mathrm{C}$ for $10 \mathrm{~min}$, centrifuged $(15000 \mathrm{~g}$, $10 \mathrm{~min}$ ), and $100 \mu \mathrm{l}$ supernatant were transferred to a polystyrene tubes evaporated to dryness in a vacuum centrifuge. The residue was dissolved in RIA buffer and VIP measured with an RIA kit from Peninsula Laboratories, Inc. (Belmont, CA, USA). The sensitivity, intra-, and interassay coefficient of the VIP assay averaged $5 \mathrm{pg}, 2.3$, and $7.1 \%$ respectively.

\section{Statistical analysis}

Data from each study were analyzed by one-way ANOVA followed by Fischers' test statistic. The effect of VIP at each cycle stage was compared by paired $t$-test. Appropriate transformations were applied when data were not normally distributed. $P$ value of 0.05 was considered to be significant.

\section{Results \\ Cyclic changes in ovarian androgen and estradiol responses to VIP}

The androgen output from ovaries obtained from various stages of estrous cycle, in the absence or presence of VIP is summarized in Fig. 1 (top panel). Ovaries incubated in the absence of VIP showed the expected cyclic changes in androgen (open bars). Maximal basal release of androgen was achieved during LP. VIP increased androgen production during DII, EP, and E but not LP. The magnitude of VIP-induced androgen release (increase over baseline) was greatest at EP (2.45-fold) than at DII (0.83) and E (0.54). Cyclic changes in estradiol output from the same ovaries are presented in the bottom panel of Fig. 1. VIP increased estradiol release from ovaries collected at DII, EP and LP, and E. Magnitude of VIP-induced estradiol release was highest during EP (2.51-fold increase over corresponding baseline) compared with other cycle stages.

Figure 2 summarizes results from studies that addressed if cyclic differences in maximal production 

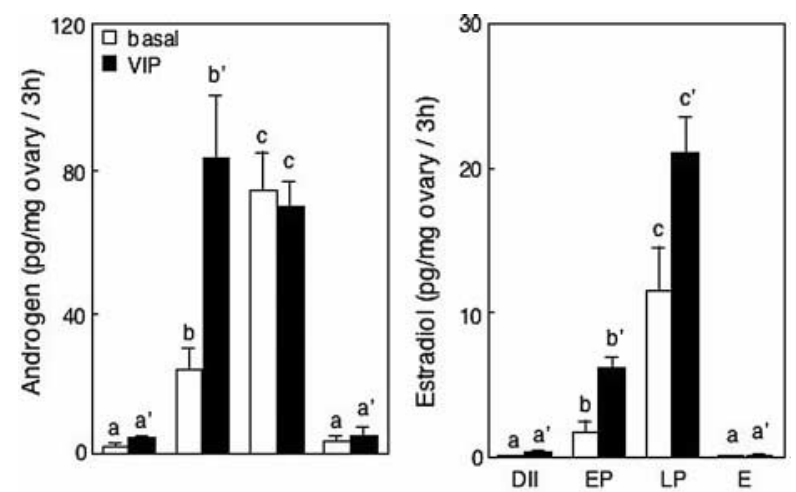

Figure 1 Androgen (left panel) and estrogen (right panel) release from ovaries collected during various stages of the rat estrous cycle both in the absence and presence of VIP. Each bar represents the mean \pm s.E.M. of five to six independent measurements. Differing letters show significant differences between cycle stages. Within cycle stage, significant VIP response is indicated by an apostrophe.

of VIP-induced estradiol release were a function of differences in availability of androgen, the estradiol precursor. In general, basal release of estradiol was higher from ovaries incubated in the presence of saturating concentrations of estradiol precursor $\Delta^{4}$ androstenedione relative to those incubated in its absence (Fig. 1 versus Fig. 2). VIP increased estradiol release at all three-cycle stages studied (LP ovaries were

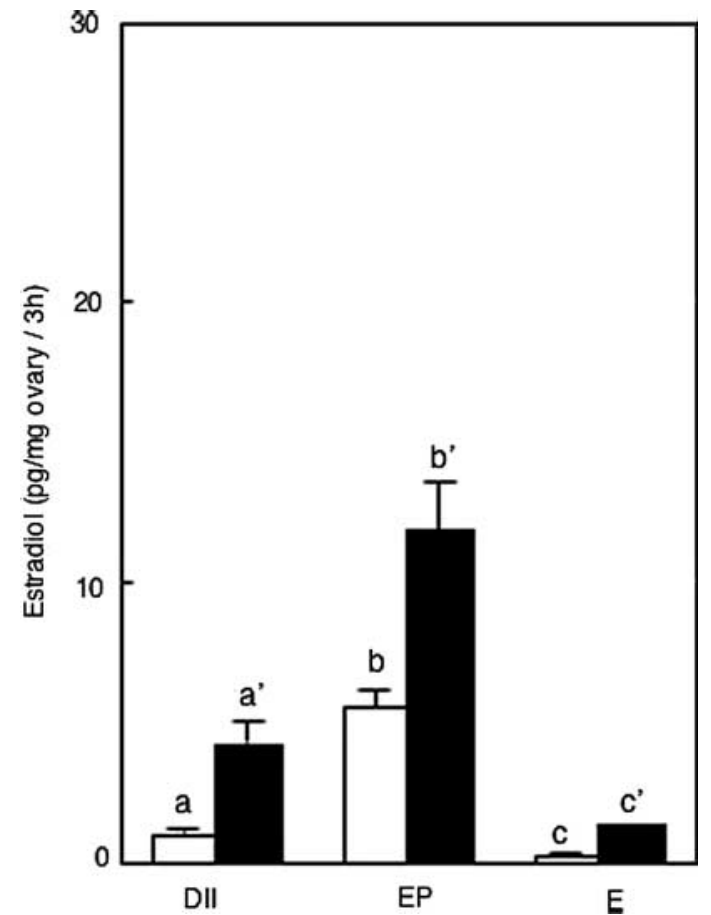

Figure 2 Estrogen release in the presence of saturating concentrations of androstenedione, from ovaries collected during three different stages of the rat estrous cycle both in the absence and presence of VIP. Each bar represents the mean \pm s.E.M. of five to six independent measurements per group. Differing letters show significant differences between cycle stages. Within cycle stage, significant VIP response is indicated by an apostrophe. inadvertently left out from this test). VIP induced a 2.92and 3.98-fold greater estradiol release from diestrus and estrus ovaries, when incubated in the presence of the precursor. This was higher than the amount released in the absence of substrate (0.83- and 0.54-fold, Fig. 1). Reflective of the increased basal estradiol release in the presence of the substrate, the magnitude of VIP-induced estradiol release from EP ovaries was lower (1.11-fold as opposed to 2.51 in the absence of substrate).

\section{Effects of VIP on androgen and estradiol release from polycystic ovaries induced by EV treatment}

Cyclic activities of five control and five EV-treated rats from which ovaries were removed for testing effects of VIP are summarized in Fig. 3 (left panel). As expected, control rats showed repetitive cycles. The EV-treatment, as reported earlier (Barria et al. 1993, Rosa-E-Silva et al. 2003), resulted in severe cycle disruption with majority remaining in persistent estrus. The ovarian morphology of two control rats during estrus and two EV-treated rats studied in parallel is shown in Fig. 3 (right panel). Ovaries of EV-treated rats exhibited characteristic polycystic ovarian morphology, as reported earlier (Barria et al. 1993, Rosa-E-Silva et al. 2003). There were no statistical differences in basal release of androgen or estrogen from control estrus and EV-treated rats (Fig. 4, top panel). VIP increased release of androgen and estrogen from ovaries of both estrus and EV-treated rats. VIP-induced release of androgen in EV-treated rats tended to be higher $(P=0.07)$ when compared with that of estrous controls. Similarly, magnitude of VIP-induced estradiol also was marginally higher $(P=0.057)$ in ovaries obtained from EV-treated rats (3.62-fold) as compared with the response of estrus rats (2.74).
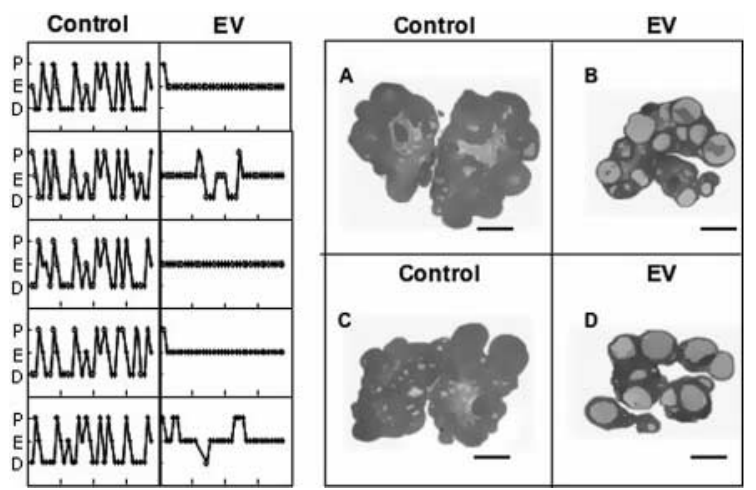

Figure 3 Effect of prepubertal administration of EV on estrous cyclicity. The panels on the left show estrous cycle patterns between 60 and 120 days of age from the five control and five EV-treated rats used in the ovarian studies (Figs 4 and 5). The panel on the right shows representative ovarian morphology from two control estrus and two EV-treated rats that were studied in parallel to confirm estrus cycle defects and development of polycystic ovarian morphology. Bar corresponds to $1 \mathrm{~mm}$. 

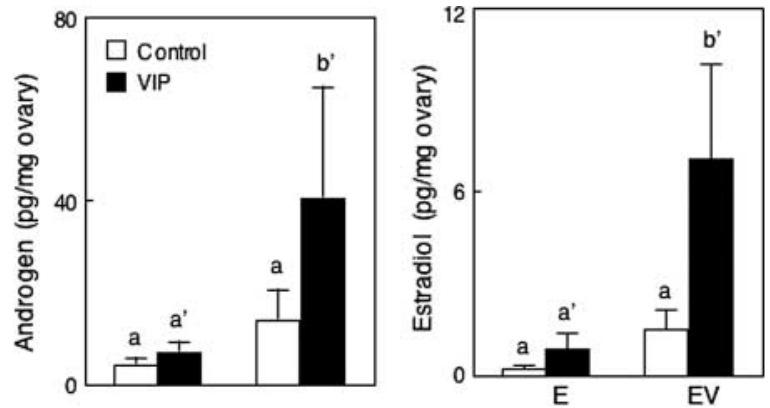

Figure 4 Effect of EV administration on ovarian secretion of androgens (right panel) and estradiol (left panel) in the absence and presence of VIP. The ovaries of control rats were obtained during estrus. Each bar represents the mean \pm s.E.M. of five independent observations per group. Differing letters show significant differences between estrus and EV-treated rats. Within groups, significant VIP response is indicated by an apostrophe.

\section{Changes in ovarian NE, VIP, and NGF concentration during the rat estrus cycle and following EV treatment}

The changes in ovarian concentrations of VIP, NE, and NGF, the growth factor responsible for growth and maintenance of autonomic nerves, during the rat estrous cycle and the EV-treated rats are summarized in Fig. 5. As we previously reported (Ferruz et al. 1991), ovarian NE concentrations were similar across estrus cycle stages but higher in EV-treated rats. Ovarian VIP concentrations varied with cycle stage, being highest during diestrus and declining during transition from diestrus to proestrus. VIP levels did not decline further during estrus. Highest levels of VIP were found in EV-treated rats. As expected, NGF levels varied during rat cycle were inversely related to that of VIP with lowest levels found during DII and increasing at EP. NGF levels of EV-treated rats were similar to that of estrous females.

\section{Discussion}

Findings from this study clearly document that VIP has stage-specific effects on ovarian steroid secretion during the rat estrous cycle and changes in ovarian VIP during
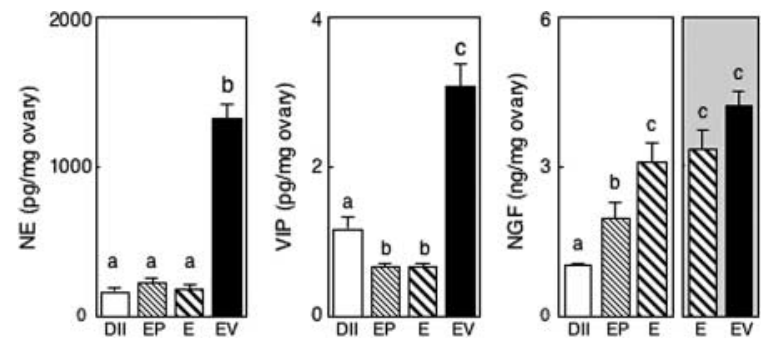

Figure 5 Changes in the concentrations of NE, VIP, and NGF in the ovary of rats obtained from various cycle stages and EV-treated rat. Each bar represents the mean \pm S.E.M. of five independent observations per group. Note for NGF measurements in EV-treated group, a second set of ovaries from estrus rats were used as controls. For each measure, differing letters indicate significant differences. the rat estrous cycle are consistent with a role for VIP in ovarian steroidogenesis. Furthermore, increased production of VIP may underlie increased ovarian capacity of EV-treated rats to produce estradiol, in the face of reduced circulating levels of FSH (reported previously; Brawer et al. 1986, Rosa-e-Silva et al. 2003).

\section{VIP and cyclic steroid production}

With the exception of pathologies where peripheral conversion could be predominant (Zayed et al. 1994), the main source of circulating estradiol is the ovary. Findings from this study document that estradiol secretion from ovarian fragments incubated in vitro parallel changes in circulating estradiol levels that occur in vivo during the rat estrous cycle (Freeman 1994), documenting the validity of the in vitro model system used. For example, the increase in estradiol secretion from ovarian fragments obtained at proestrus paralleled the increase in steroid biosynthetic capacity of the ovary that occurs during the periovulatory period in rats that is responsible for the generation of the LH surge (Freeman 1994). The changes in cyclic androgen production from ovarian fragments incubated in vitro are also consistent with androgen serving as a precursor for estrogen biosynthesis. In support of this, maximal androgen release occurred from ovarian fragments concomitantly with the maximal increase in estradiol production, which occurred from ovaries obtained during EP.

Consistent with a stimulatory role for VIP in estradiol production, the LP increase in basal estradiol production was preceded by (1) an increase in ovarian levels of VIP at DII and (2) increased capacity of VIP to maximally induce estradiol release. The effect of VIP in stimulating androgen secretion from DII, EP, and E ovaries may be reflective of the ability of VIP to increase P450 SSC enzyme complex, the rate-limiting enzyme in $\mathrm{P}_{4}$ biosynthesis (Trzeciak et al. 1986, Johnson et al. 1994). The maximal VIP-induced release of androgen secretion occurred from the EP ovaries, the cycle stage where the activity of SSC complex is limiting to induce a large spontaneous increase in ovarian androgen release (Freeman 1994). Consistent with this concept, VIP did not affect androgen release from ovarian fragments of LP rats, the cycle stage where maximal activity of SSC complex is present (Ahmed et al. 1986).

VIP's ability to stimulate estradiol biosynthesis throughout the estrous cycle, albeit in cycle specific manner, may also be related to its ability to stimulate P450 aromatase enzyme complex (George \& Ojeda 1987). Time-course studies carried out incubating rat ovaries for 1, 2, and $3 \mathrm{~h}$ produced a linear increase in estradiol secretion as a function of increasing incubation time (not shown). This combined with the fact that steroids are secreted as soon as they are biosynthesized and cyclic changes in ovarian estradiol production are mimicked in in vitro incubation studies suggest that the 
response is specific and likely to involve increased aromatase activity.

Increased basal release of estradiol from ovaries obtained from all stages of the cycle following inclusion of saturating concentrations of androstenedione suggests that, under the in vitro conditions used, the levels of aromatase may not be limiting and the rate-limiting step may be the availability of estrogen substrate, androgen. This also implies that the capacity of VIP to stimulate estradiol production may be secondary to increased production of androgen and availability of aromatase. The changes in ovarian levels of VIP are also consistent with its stimulatory role in estradiol biosynthesis. The marked decrease in ovarian VIP content between DII and EP may reflect increased release of the peptide from the ovary.

The effects of VIP in increasing estradiol secretion may be mediated through increased aromatase biosynthesis or increase in specific activity of the aromatase enzyme, both of which are not mutually exclusive. An increase in aromatase activity is predicted by the potentiating effect of VIP on estradiol secretion during LP, when there is no effect of VIP on androgen secretion. An increase in specific activity of the aromatase enzyme or increased availability of aromatase is predicted by VIP-induced increase in estradiol secretion under saturating concentration of $\Delta^{4}$-androstenedione, the substrate for estradiol biosynthesis. Earlier studies have provided evidence that VIP is capable of inducing aromatase activity from immature follicles prior to their acquisition of FSH receptors (Mayerhofer et al. 1997). A possibility to consider is that VIP induction of estradiol release during the estrus cycle may also come from immature follicles. If true, this would suggest that VIP plays a complementary role to that of $\mathrm{FSH}$, the primary mediator of estradiol biosynthesis, in determining the magnitude of estradiol increase under varying physiologic conditions. A role for VIP/pituitary adenylate cyclase-activating peptide (PACAP) in follicular development has also been recently proposed from studies in mouse (Cecconi et al. 2004). Since both peptides occupy the different VIP/PACAP receptor subtypes with the same affinity (Igarashi et al. 2002), it is difficult to distinguish specific effects of VIP on the receptor population from PACAP effects. As such no measures of VIP receptors were undertaken.

\section{Changes in ovarian VIP}

VIP containing nerve fibers within the ovary have been localized in sympathetic nerves fibers originating in paravertebral ganglia that travel through the superior ovarian nerve to the ovary (Klein \& Burden 1988). In the ovary, these fibers were found to be associated with blood vessels, ovarian stroma, and primordial and antral follicles (Dees et al. 1986, Klein \& Burden 1988). Evidence exists to document the presence of VIP mRNA in the rat ovary (Gozes \& Tsafriri 1986). Later studies with bovine ovaries suggest granulosa cells as also a probable site of biosynthesis of VIP (Hulshof et al. 1994).

Since the cyclic intraovarian VIP changes occurred every 4 days, it is unclear to what extent changes in VIP transcription/translation occurring at the cell body of VIPergic neurons located at the celiac ganglion account for these changes. A possibility to consider is that at least a fraction of the VIP regulation of estradiol release involves changes in locally produced VIP (Gozes \& Tsafriri 1986, Hulshof et al. 1994). From a regulatory perspective, it is difficult to compare levels of VIP used (10-6 M; $\sim 3700 \mathrm{ng} / \mathrm{ml})$ in the in vitro studies with ovarian tissue levels of VIP ( $\sim 150 \mathrm{pg} /$ ovary). Considering that nerve terminals and granulosa cells produce VIP, VIP levels must be several orders of magnitude higher at the granulosa cell level (granulosa compartment constitutes a very small portion of the whole ovary).

The inverse relationship between intraovarian NGF and VIP throughout the estrous cycle and the fact that NGF blocks the expression of VIP mRNA in many tissues (Zigmond \& Sun 1997, Brodski et al. 2000, Helke \& Verdier-Pinard 2000) suggest that locally produced VIP may be in turn regulated by neural-derived NGF. If the intraovarian VIPergic system becomes active between DII and PE, when follicular selection and dominance is achieved, it is suggestive of functional complementarities between peptidergic input and noradrenergic innervation similar to that demonstrated previously for activation of NE system between PE and E (Ferruz et al. 1991). The decrease in ovarian VIP between DII and EP could be the result of depletion from increased release of the neuropeptide before proestrus into the ovary. This appears highly likely, because neuropeptides (source of VIP content) are not synthesized at nerve terminals that innervate the ovary (Lundberg 1996). The low concentrations of the peptide precluded us from determining the release pattern of the peptide during the different stages of the estrous cycle.

\section{VIP- and EV-induced polycystic ovaries}

In addition to the role VIP plays in regulating cyclic androgen and estradiol production, the tendency of VIP to increase androgen and estradiol production from ovaries of EV-treated rats compared with ovaries from estrus rats suggest a potential role for VIP in the development of the ovarian pathology in this model. Since granulosa cell compartment is also the site for VIP biosynthesis (Hulshof et al. 1994) and the ovarian cysts seen in EV-treated rats have reduced granulosa cell layers (Brawer et al. 1986, Lara et al. 2000), the similar intraovarian level of VIP in EV-treated and estrus rats may be reflective of increased innervation of the polycystic ovaries of the EV-treated rats. However, before establishing a role for VIP in the initiation of ovarian pathologies, detailed studies at individual follicle levels are required. 
Earlier finding that VIP cannot induce luteinizing hormone receptors (Davoren \& Hsueh 1985) is consistent with failure of cystic follicles of EV-treated rats to achieve dominance. If a role for VIP in induction of polycystic ovaries can be established, these findings may have relevance to the follicular arrest seen in women with PCOS. EV-treated rats share several attributes of women with PCOS, namely anovulation, polycystic ovarian morphology, and a tendency for increased androgen production (Rotterdam consensus 2004).

In summary, cyclic changes in ovarian VIP concentrations and the capacity of VIP to increase androgen and/or estradiol secretion during the estrous cycle and from polycystic ovaries induced by EV strongly suggest a physiological role for this peptide in ovarian steroid biosynthesis.

\section{Acknowledgements}

Supported by Fondo Nacional de Ciencias 102-0581. The authors declare that there is no conflict of interest that would prejudice the impartiality of this scientific work.

\section{References}

Adashi EY \& Hsueh AJ 1982 Estrogens augment the stimulation of ovarian aromatase activity by follicle-stimulating hormone in cultured rat granulosa cells. Journal of Biological Chemistry 257 6077-6083.

Advis JP \& Ojeda SR 1978 Hyperprolactinemia-induced precocious puberty in the female rat: ovarian site of action. Endocrinology $\mathbf{1 0 3}$ 924-935.

Ahmed CE, Dees WL \& Ojeda SR 1986 The immature rat ovary is innervated by vasoactive intestinal peptide (VIP)-containing fibers and responds to VIP with steroid secretion. Endocrinology 118 1682-1689.

Barria A, Leyton V, Ojeda SR \& Lara HE 1993 Ovarian steroidal response to gonadotropins and beta-adrenergic stimulation is enhanced in polycystic ovary syndrome: role of sympathetic innervation. Endocrinology 133 2696-2703.

Brawer JR, Munoz J \& Farooki R 1986 Development of the polycystic ovarian condition (PCO) in the estradiol valerate-treated rat. Biology of Reproduction 35 647-655.

Brodski C, Schnurch H \& Dechant G 2000 Neurotrophin-3 promotes the cholinergic differentiation of sympathetic neurons. PNAS 97 9683-9688.

Burden HW 1998 The adrenergic innervation of mammalian ovaries. In Catecholamines as Hormones Regulators, pp 261-278. Eds N BenJonathan, JM Bahr \& RI Weiner. New York: Serono Symposia Publications, Raven Press.

Cecconi S, Rossi G, Barberi M, Scaldaferri L \& Canipari R 2004 Effect of pituitary adenylate cyclase-activating polypeptide and vasoactive intestinal polypeptide on mouse preantral follicle development in vitro. Endocrinology 145 2071-2079.

Davoren JB \& Hsueh AJW 1985 Vasoactive intestinal peptide: a novel stimulator of steroidogenesis by cultured granulosa cells. Biology of Reproduction 33 37-52.

Dees WL, Ahmed CE \& Ojeda SR 1986 Substance P- and vasoactive intestinal peptide-containing fibers reach the ovary by independent routes. Endocrinology $119638-641$.
Dissen GA, Lara HE, Leyton V, Paredes A, Hill DF, Costa ME, Martinez Serrano A \& Ojeda SR 2000 Intraovarian excess of nerve growth factor increases androgen secretion and disrupts estrous cyclicity in the rat. Endocrinology 141 1073-1082.

Dorfman M, Fiedler JL, Arancibia S \& Lara HE 2003 Chronic intermittent cold stress activates ovarian sympathetic nerves and modifies ovarian follicular development in the rat. Biology of Reproduction 68 2038-2043.

England BG, Niswender GD \& Midgley AR Jr 1974 Radioimmunoassay of estradiol-17ß without chromatography. Journal of Clinical Endocrinology and Metabolism 38 42-50.

Ferruz J, Barria A, Galleguillos X \& Lara HE 1991 Release of norepinephrine from the rat ovary: local modulation by gonadotropins. Biology of Reproduction 45 592-597.

Fredericks CM, Lundquist LE, Mathur RS, Ashton SH \& Landgreve SC 1983 Effects of vasoactive intestinal polypeptide upon ovarian steroids, ovum transport and fertility in the rabbit. Biology of Reproduction 28 1052-1060.

Freeman MC 1994 The neuroendocrine control of the ovarian cycle in the rat. In The Physiology of Reproduction, pp 613-658. Eds E Knobil \& JD Neill. New York: Raven Press.

George FW \& Ojeda SR 1987 Vasoactive intestinal peptide enhances aromatase activity in the neonatal rat ovary before development of primary follicles or responsiveness to follicle-stimulating hormone. PNAS 84 5803-5807.

Gozes I \& Tsafriri A 1986 Detection of vasoactive intestinal polypeptide-encoding messenger ribonucleic acid in the rat ovaries. Endocrinology 119 2606-2610.

Helke CJ \& Verdier-Pinard D 2000 Neurotrophins alter the numbers of neurotransmitter-ir mature vagal/glossopharyngeal visceral afferent neurons in vitro. Brain Research 884 206-212.

Hillier SG \& De Zwart FA 1981 Evidence that granulose cell aromatase induction/activation by follicle-stimulating hormone is an androgen receptor-regulated process in-vitro. Endocrinology 109 1303-1305.

Hulshof SC, Dijkstra G, Van der Beek EM, Bevers MM, Figueiredo JR, Beckers JF \& Van der Hurk R 1994 Immunocytochemical localization of vasoactive intestinal polypeptide and neuropeptide $\mathrm{Y}$ in the bovine ovary. Biology of Reproduction 50 553-560.

Igarashi H, Ito T, Pradhan TK, Mantey SA, Hou W, Coy D \& Jensen RT 2002 Elucidation of the vasoactive intestinal peptide pharmacophore for $\mathrm{VPAC}_{2}$ receptors in human and rat and comparison to the pharmacophore for $\mathrm{VPAC}_{1}$ receptors. Journal of Pharmacology and Experimental Therapeutics 303 445-460.

Johnson AL, Gibney JA \& Malamed S 1994 Vasoactive intestinal polypeptide-induced expression of cytochrome P450 cholesterol side-chain cleavage and 17 alpha-hydroxylase enzyme activity in hen granulosa cells. Biology of Reproduction 51 327-333.

Klein CM \& Burden HW 1988 Substance P (SP) and vasoactive intestinal polypeptide (VIP)-immunoreactive nerve fibers in relation to ovarian postganglionic perikarya in para and prevertebral ganglia: evidence from combined retrograde tracing and immunocytochemistry. Cell and Tissue Research 252 403-410.

Lara HE, Ferruz JL, Luza S, Bustamante DA, Borges Y \& Ojeda SR 1993 Activation of ovarian sympathetic nerves in polycystic ovary syndrome. Endocrinology 133 2690-2695.

Lara HE, Dissen GA, Leyton V, Paredes A, Fuenzalida H, Fiedler JF \& Ojeda SR 2000 An increased intraovarian synthesis of nerve growth factor and its low affinity receptor is a principal component of steroid-induced polycystic ovary in the rat. Endocrinology 141 1059-1072.

Lundberg JM 1996 Pharmacology of cotransmission in the autonomic nervous system: integrative aspects on amines, neuropeptides, adenosine triphosphate, aminoacids and nitric oxide. Pharmacological Reviews 48 113-178.

Mayerhofer A, Dissen GA, Costa ME \& Ojeda SR 1997 A role for neurotransmitters in early follicular development: induction of functional follicle-stimulating hormone receptors in newly formed follicles of the rat ovary. Endocrinology 138 3320-3329. 
Ojeda SR \& Lara HE 1989 The role of the sympathetic nervous system in the regulation of ovarian function. In The Menstrual Cycle and its Disorders, pp 26-33. Eds KM Pirke \& U Schweiger. Berlin: SpringerVerlag.

Ojeda SR \& Urbanski HF 1994 Puberty in the Rat. In The Physiology of Reproduction, pp 362-409. Eds E Knobil \& JD Neill. New York: Raven Press.

Ojeda SR, Lara H \& Ahmed CE 1989 Potential relevance of vasoactive intestinal peptide to ovarian physiology. Seminars in Reproductive Endocrinology 7 52-60.

Rosa-e-Silva AR, Guimaraes MA, Padmanabhan V \& Lara HE 2003 Prepubertal administration of estradiol valerate programs anovulation and polycystic ovarian morphology during adult life in the rat. Role of sympathetic innervation. Endocrinology 144 4289-4297.

Rotterdam ESHRE/ASRM-sponsored PCOS consensus workshop group 2004 Revised 2003 consensus on diagnostic criteria and long-term health risks related to polycystic ovary syndrome (PCOS). Human Reproduction 19 41-47.
Trzeciak WH, Waterman MR, Simpson ER \& Ojeda SR 1986 Vasoactive intestinal peptide induces the synthesis of the cholesterol side-chain cleavage enzyme complex in cultured rat ovarian granulosa cells. PNAS 83 7490-7494.

Zayed A, Stock JL, Liepman MK, Wollin M \& Longcope C 1994 Feminization as a result of both peripheral conversion of androgens and direct estrogen production from adrenocortical carcinoma. Journal of Endocrinological Investigation 17 275-278.

Zigmond RE \& Sun Y 1997 Regulation of neuropeptide expression in sympathetic neurons. Paracrine and retrograde influences. Annals of the New York Academy of Sciences 814 181-197.

Received 4 April 2006

First decision 5 June 2006

Revised manuscript received 7 November 2006

Accepted 15 November 2006 